\title{
Research on Trumpchi Brand DNA Based on Visual Feature Analysis
}

\author{
Yu Chen \\ School of Design \\ South China University of Technology \\ Guangzhou, China
}

\author{
Mengming Lu \\ School of Design \\ South China University of Technology \\ Guangzhou, China
}

\begin{abstract}
Based on the complexity of automobile appearance modeling, the products of Trumpchi brand's main product lineage are taken as the analysis object and the key modeling lines in automobile modeling design are extracted for visual semantic analysis from the perspective of visual features and semantic characteristics. This paper reveals the overall change law of Trumpchi brand DNA and summarizes the inheritance and evolution of Trumpchi brand DNA.
\end{abstract}

Keywords—visual feature; characteristic line; brand DNA

\section{INTRODUCTION}

The brand is an indirect expression of the company's goals and core values. The visual features of the product represent the styling information recognized by the vision, which is the embodiment of the brand goal and brand characteristics. The visual features of the product are mainly composed of styling features and visual semantics. In the field of automotive brand, from the concept of automobile brand to the iconic characteristics of major automobile brands, the styling features and visual semantics of automobiles become the main method to distinguish automobile brands. The user has formed a brand impression with visual features as the main identification method to select and identify the brand. The establishment of a car brand has certain brand development rules in the inheritance and evolution of visual features. This paper analyzes the visual features and the evolution of visual semantics to find out the rules of the family design of Trumpchi brand.

\section{VISUAL FEATURES AND BRAND DNA}

"特征"(Chinese character) is "Feature" or "characteristic" in English. Literally, it means that the character of one thing is different from that of other things. For the product form, it mainly refers to the unique or significant part of the form. It can be said that it is significant and distinctive morphological characteristic [1]. Usually, when contacting new products, the first factor that affects human vision is the appearance of the product, and the second is the perception of the visual semantics produced by the appearance of the product.
In the car styling, the most intuitive feeling people have comes from the car styling feature line, and the followed is the product structure theory based on the point, line and surface. The overall styling gives people a semantic feature feeling. For example, the profile contour feature of front face of Wuling motors is regarded as "Pig head", which gives people a skinny, honest and simple feeling [2]. The cheetah image of Leopaard car gives people a speed and light feeling. Therefore, the visual image derived from the car characteristic line will affect people's perception of the product brand image.

For the automobile brand, the product shape formed by the key characteristic lines of the automobile styling characteristics, the automobile materialized design image and the brand LOGO mutually map each other. It will form a brand image in the consumer's mind. Then, it will form the automobile brand DNA. At the same time, car styling has become an important indicator factor for consumers to buy cars.

\section{THE DEVELOPMENT HISTORY OF TRUMPCHI AUTOMOBILE BRAND AND THE SELECTION OF RESEARCH OBJECTS}

Trumpchi brand was established in 2008 and has ten years of history. As shown in "Fig. 1", the main development history of Trumpchi brand is divided into Trumpchi 1.0 and Trumpchi 2.0. From September 13, 2010, Trumpchi released the first car named GA5, which announced the birth of China's own auto brand. Subsequently, Trumpchi launched the GS5, the first SUV-type car, and began to grasp the viability in the market. In 2013, GA3 of the first compact modeling was released, and Trumpchi has the strength to impact the main market of the car. From the release of the GA3S, the family-style design of Trumpchi automobile brand began to show up. Later, Trumpchi Automobile continuously released the GS5 Super, GA5REV, GA6, GS4, GA8 and other cars. Trumpchi brand is remembered by the market. Gradually, it has form the brand identity in the hearts of consumers. 


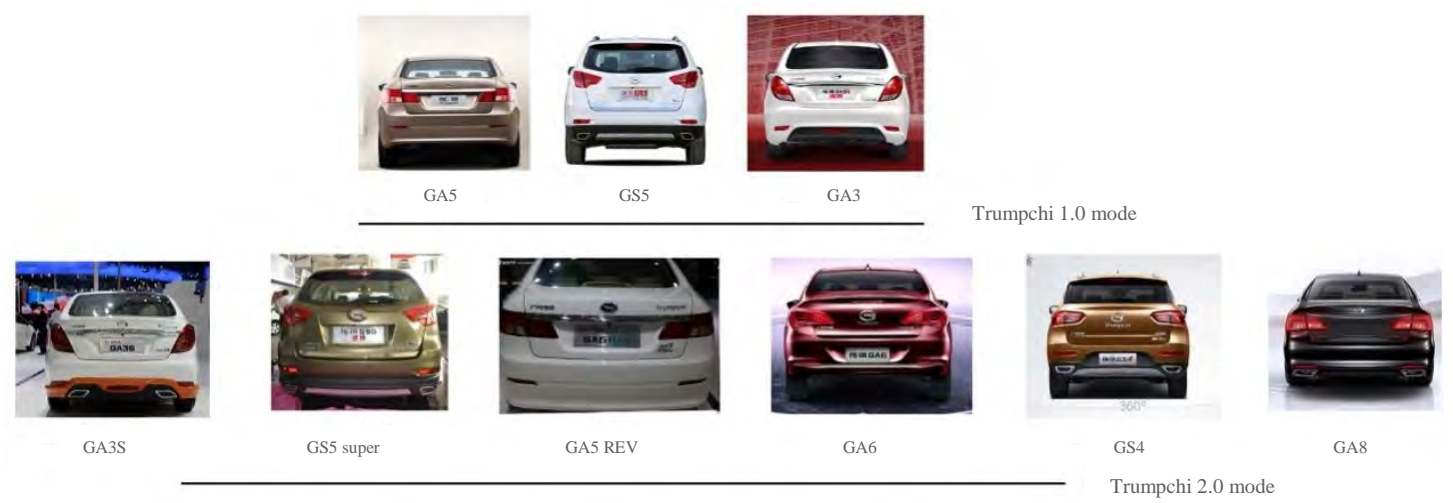

Fig. 1. The development line of Trumpchi.

Trumpchi brand has ten years of the development history. Through the development history of Trumpchi brand, the products of the main product lineages of Trumpchi brand are selected for feature analysis and feature refinement. The product modeling characteristics in the development process of Trumpchi brand are refined and analyzed. We can find the overall change law of the brand DNA.

\section{CALIBRATION AND ANALYSIS OF TRUMPCHI STYLING FEATURES}

This paper intends to select 9 representative cars in the important development period of Trumpchi brand. At the same time, in view of the complexity of automobile modeling, it is proposed to select the feature lines of front face shape, tail shape and side shape as the modeling unit.

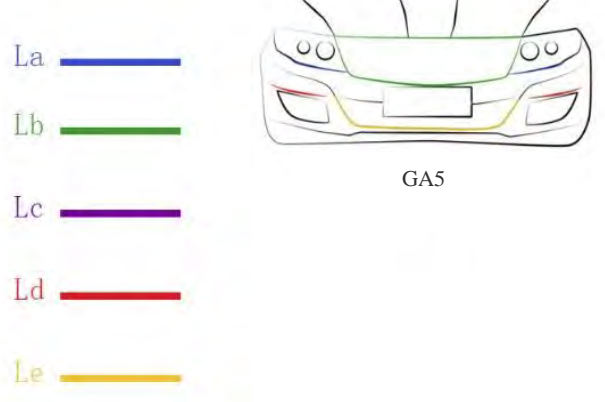

Fig. 2. The front face feature line of Trumpchi GA series.

The extraction results of the GA series show that La line of the headlights and Lb line of the grille are getting farther and farther, and the angle between the La line and the horizontal line shows GA3 horizon $<\mathrm{GA} 3<\mathrm{GA} 5<\mathrm{GA} 6<\mathrm{GA} 8$. In term of the inclination, GA8 inclination is greater, which brings prople a stronger sense of speed and fierceness. The $\mathrm{Lb}$ wire loop of grille gradually becomes rounded. The whole body is gradually inclined upward, which has a
Image source: painted by the autho The continuity and the evolution of family characteristics of Trumpchi brand are explored.

\section{A. Front Face Modeling Features}

In the front face modeling, feature lines are extracted for important features such as the grille, the headlights, the fog lights, and the hood line molding line in the front face shape. Through the feature line calibration method in Tan Zhengyu's "The styling feature calibration and recognition pattern construction of automobile brand" [2], the extracted front face contour feature lines are named and text-calibrated. As shown in "Fig. 2" and "Fig. 3", the key control lines (La, $\mathrm{Lb}, \mathrm{Lc}, \mathrm{Ld}, \mathrm{Le}, \mathrm{Lf}, \mathrm{Lg}, \mathrm{Lh})$ together form the change of contour feature line of the front face shape.

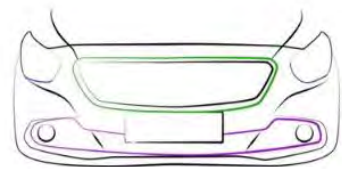

GA3

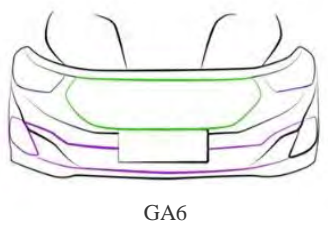

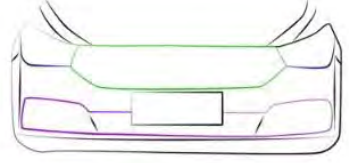

GA3 horizon

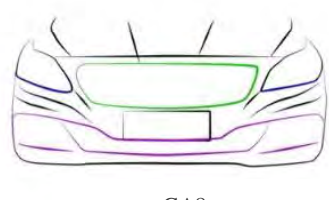

GA8 a. Image source: painted by the author stronger sense of stability and a sense of leaning forward. The Ld line and Le line of fog lights gradually merge into a whole (Lc line), which eventually evolves into Lc feature of the fog light. Both ends of the Lc are gradually inclined upward, forming a sense of leaning forward. It gives people a sense of speed and sharpness. The changing trends of the three lines of $\mathrm{La}, \mathrm{Lb}$ and $\mathrm{Lc}$ jointly constructed the dynamic and fierce feeling of the front face of the car. 

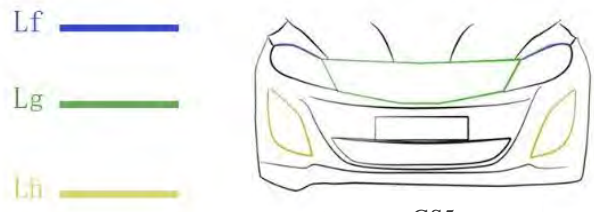

GS5

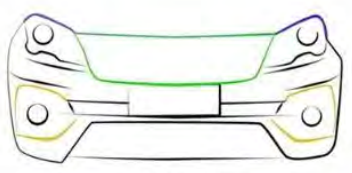

GS5 super

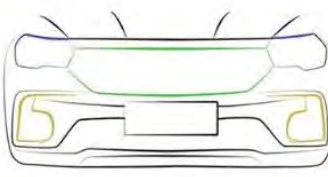

GS4

Fig. 3. The front face feature line of Trumpchi GS series.

The extraction results of the GS series show that the Lf line of the headlights and the horizontal hood line above the $\mathrm{Lg}$ line of the grille are gradually integrated into one body, which has a more obvious sense of stability. The Lg line of the grille gradually changes from a pentagon to a hexagon,

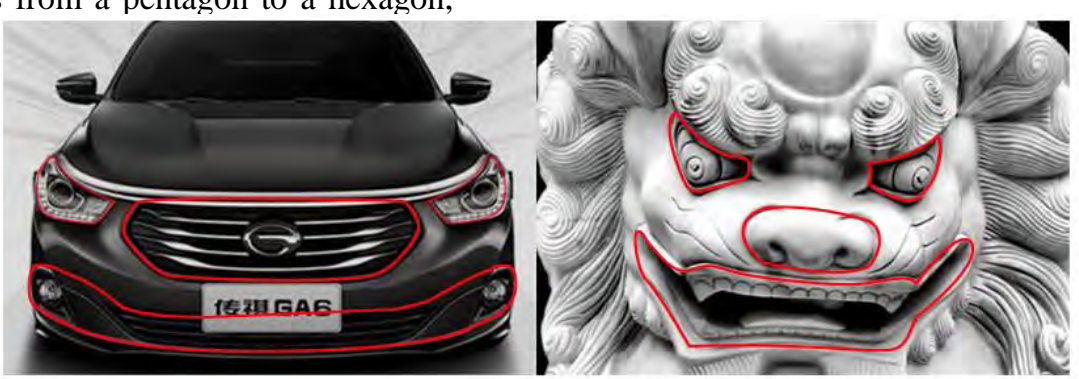

Fig. 4. Formation of stone lion's facial makeup modeling.

The GA series is different from the GS series. In the process of constant adjustment and evolution of the feature line, the original and recognized front face design of Trumpchi brand is highlighted. The front face design of Trumpchi is based on the Chinese aesthetic and the tradition of placing stone lions in front of the door to facilitate the avoidance of misfortunes. The fierce image of the bionic stone lions is integrated with the shape of the front to make the abstract combination. The pattern with the facial features is applied to the front face design. The front face feature helps to form formulaic family DNA with the characteristic of the times. In the car modeling, through the extension of the headlamp, the amplification of the intake grille, and the use of the overall curve of the fog lamp, people will feel that it is fierce and powerful, giving a strong visual impact. As shown in "Fig. 4". which has integrated with the headlamp characteristic lines into the whole. The Lh feature line of the fog light gradually changes to meet the shape of the front face, and the front face shape is more and more recognizable.
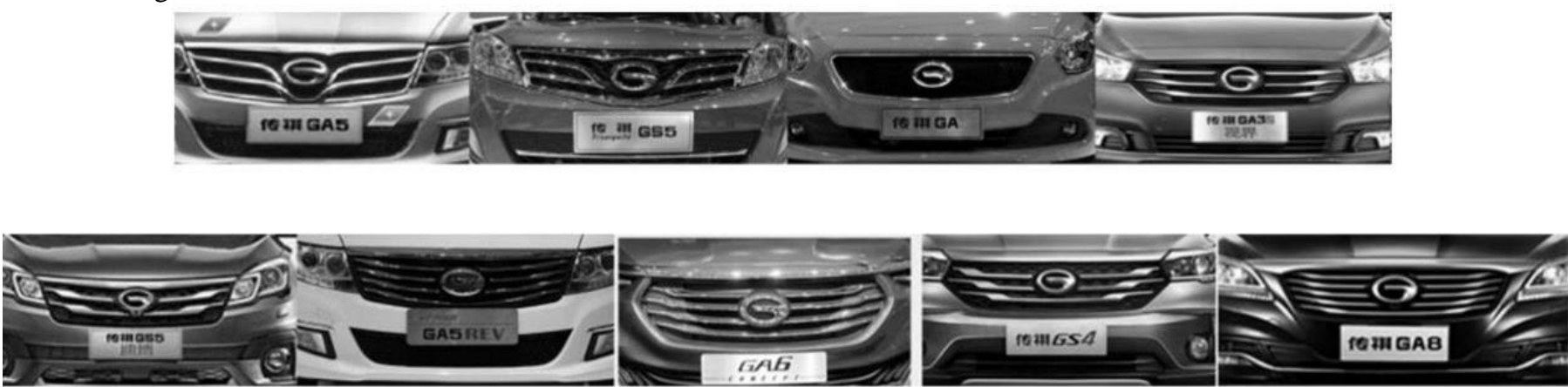

Fig. 5. The formation of Flying Dynamics family DNA. 


\section{B. Tail Shape Features}

In the tail shape, the characteristic line of the taillights is an important feature to describe the shape of the car. The feature lines of the tail shape of the car are extracted and labeled as $\mathrm{Li}$, as shown in "Fig. 6".

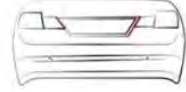

GA5

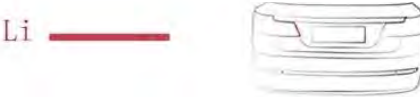

GA5 REV

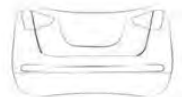

GS5
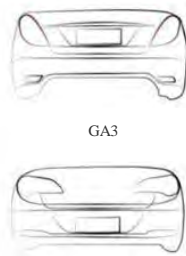

GA6

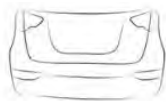

GS5 super

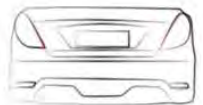

GA3 horizon
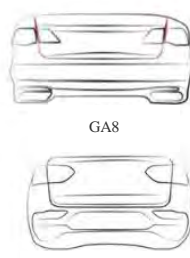

Fig. 6. The tail feature line of GA and GS series of Trumpchi brand

The extraction results show that the angle between the $\mathrm{Li}$ line marked by the red line and the vertical direction is GS5 $>$ GA3 horizon $>$ GA3 $>$ GA6 $>$ GA8, GS5>GS5 Super $>$ GS4, in which the angle between GA8 and GS4 is the smallest, and the performance is more and more stable. The taillights and tailpipes are the details of the changing trend. In the process of abstract integration of feature lines, they gradually evolve into "G"-shape lights. And this light has carried out much Trumpchi logo genes. The overall shape increasingly becomes a entirety, which echoes with the front face.

Lj

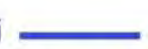

Lk

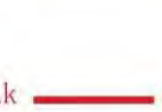

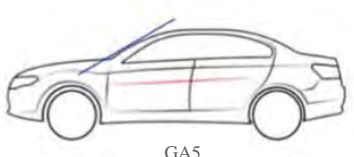

GA5

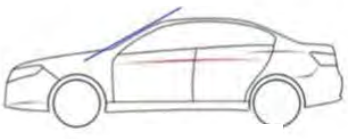

GA5REV

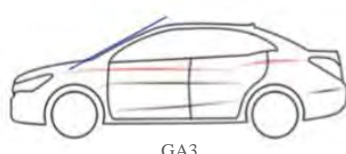

GA3

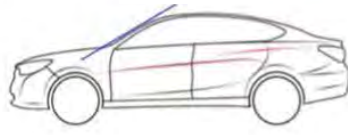

GA6

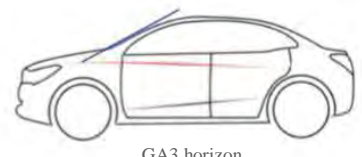

GA3 horizon

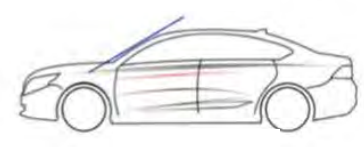

GA8

Fig. 7. The side face feature line of Trumpchi GA series.

The extraction results of the GA series show that the angle formed by the front window line, $\mathrm{Lj}$ and the horizontal line does not change much. The overall relationship is GA8 $<$ GA6 $<$ GA5REV $<$ GA3 $=$ GA3 horizon $<$ GA5, however, the overall performance of car roof is more inclined. Visually, the direction of leaning forward is clear. The angle between the waist line Lk and the horizontal line is as the following:
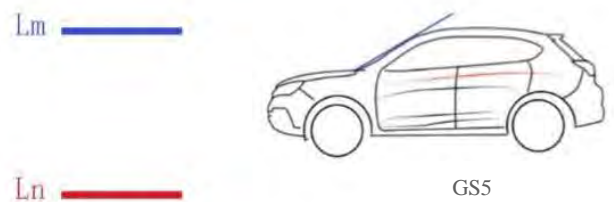

GS5

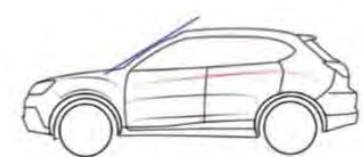

GS5 super

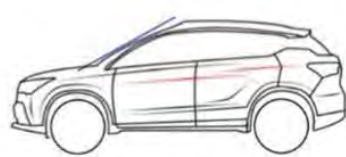

GS4

Fig. 8. The side face feature line of Trumpchi GS series. 
According to the extraction results of the GS series, the angle between the front window line Lm and and the horizontal line, and the angle between the waist line Ln and the horizontal line are basically the same. However, GS4 split waist line is higher, and the sports style is stronger.
The feature line extraction of the hood line, the front window line and the waist line of the car is performed as shown in "Fig. 9".
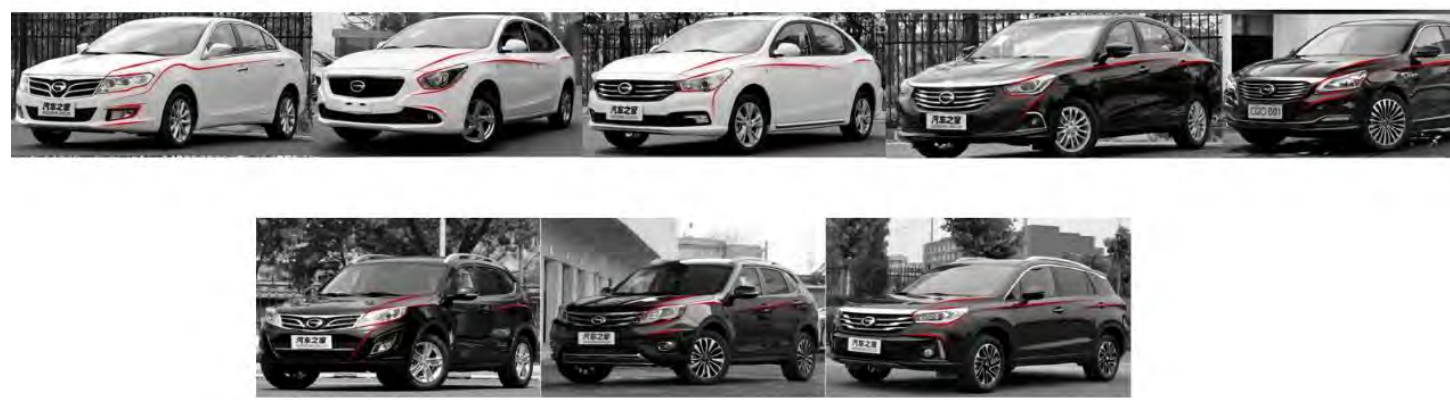

Fig. 9. Three-dimensional angle feature line of Trumpchi.

The extraction results show that the docking among the hood line, the front window line and the waist line is more and more smooth, and the overall continuity is getting stronger and stronger. The overall car is speedier, more flexible and conveys a powerful force.

\section{The Analysis of Trumpchi Styling Trend}

Based on the analysis of the trend of shape feature line variation of Trumpchi GA series and GS series modelings and the clue of generating semantic feature, we can know whether the analysis of the overall shape is consistent. It can be found that Trumpchi takes the facial stone lion image and the bionic design of the eagle's spirit and strength, forming the core family DNA design. Secondly, with the description of the change trend of the front face feature line, the side face feature line and the tail feature line of the brand shape, the description of the visual semantic change, and the description of the feature scene, Trumpchi has completed the overall calibration of the brand feature. It can be found that Trumpchi Automobile mainly focuses on stability and agility in visual characteristics, fully reflecting the family DNA design of stone lion and the eagle.

\section{CONCLUSION}

The shaping of China's own brands is destined to be a long and arduous process, which includes the exploration of the cultural positioning of Chinese automobile brands, as well as the exploration of the aesthetic needs of Chinese people. This paper takes the evolution trend and visual semantic trend of visual feature structure of Trumpchi automobile brand as a clue to explore the overall change law of Trumpchi brand DNA, which is the result of the interaction of visual features and visual feature semantics. Trumpchi is a latecomer in the Chinese market. It is prominent among many automobile brands and recognized by the consumers. The development of family-type DNA plays a major role, which provides great references for the self-recognition and design of domestic auto brands. a. Image source: painted by the author

\section{REFERENCES}

[1] Zhao Danhua, Zhao Jianghong. Styling characteristics and feature line of automobiles [J]. Packaging Engineering, 2007(03): 115-117.

[2] Tan Zhengyu, Wang Zhen. The styling feature calibration and recognition pattern construction of automobile brand $[\mathrm{J}]$. Decoration, 2013(04): 119-120.

[3] Hu Weifeng, Zhao Jianghong, Zhao Danhua. Study on the image of automobile modeling based on styling feature line[J].China Mechanical Engineering, 2009, 20(04): 496-500.

[4] Tan Zhengyu, Dong Ruyue, Zhao Jianghong, Xing Shaowei Research on design of self-owned brand SUV based on styling features[J]. Packaging Engineering, 2012, 33(24): 32-36.

[5] Ke Shanjun, Liu Hongjie. Research on brand attribute evaluation of automobile modeling[J]. Journal of Chongqing University of Technology (Natural Science), 2016, 30(07): 26-29.

[6] Liang Qiao, Zhao Jianghong. The clues of automobile brand modeling based on visual and semantic features[J]. Packaging Engineering, 2014, 35(08): 26-29+34.

[7] Wang Zhen, Tan Zhengyu. Research on automobile modeling features based on holistic cognition[J]. Packaging Engineering, 2013, 34(24): 51-54.

[8] Xu Shihu, Wu Han. The Enlightenment of Kia Auto's facial modeling on Chinese automobile design[J]. Packaging Engineering, 2012, 33(16):47-51.

[9] Zhao Danhua, He Renke, Tan Hao, Li Ran. Semantic acquisition and expression of automobile brand styling [J]. Packaging Engineering, 2013, 34(10): 27-30+61.

[10] Yao Jun, Jin Ran, Han Shudong, Fu Bo. On the inheritance and innovation of brand genes in automobile design [J]. Packaging Engineering, 2012, 33(24): 37-41.

[11] Ke Shanjun, Wei Ying, Cheng Zhenbo. Research on brand attribute characteristics of Hyundai automobile modeling[J]. Packaging Engineering, 2017, 38(02): 110-114.

[12] Qin Yan. Analysis of the design of automobile headlamps based on brand design[J]. Packaging Engineering, 2015, 36(18): 87-91.

[13] Yu Conggang, Zhu Lusha. Research on mapping from brand features to product modeling[J]. Packaging Engineering, 2014, 35(16): 1-4 and 9. 